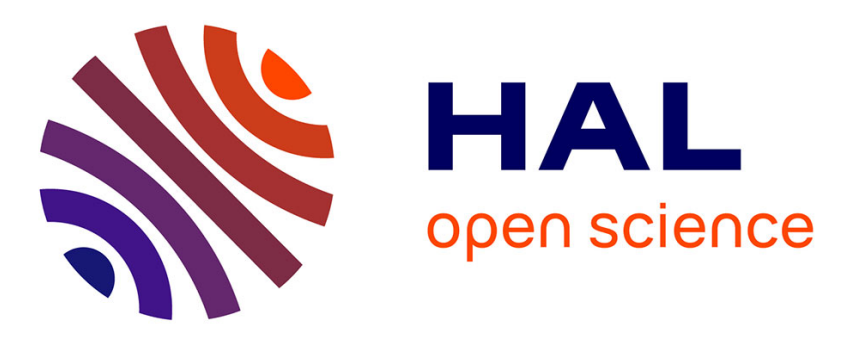

\title{
Review and evaluation of commonly-implemented background subtraction algorithms
}

Yannick Benezeth, Pierre-Marc Jodoin, Bruno Emile, Hélène Laurent, Christophe Rosenberger

\section{- To cite this version:}

Yannick Benezeth, Pierre-Marc Jodoin, Bruno Emile, Hélène Laurent, Christophe Rosenberger. Review and evaluation of commonly-implemented background subtraction algorithms. International Conference on Pattern Recognition, Dec 2008, Tampa, United States. 10.1109/ICPR.2008.4760998 . inria-00545518

\section{HAL Id: inria-00545518 \\ https://hal.inria.fr/inria-00545518}

Submitted on 16 Oct 2012

HAL is a multi-disciplinary open access archive for the deposit and dissemination of scientific research documents, whether they are published or not. The documents may come from teaching and research institutions in France or abroad, or from public or private research centers.
L'archive ouverte pluridisciplinaire HAL, est destinée au dépôt et à la diffusion de documents scientifiques de niveau recherche, publiés ou non, émanant des établissements d'enseignement et de recherche français ou étrangers, des laboratoires publics ou privés. 


\title{
Review and Evaluation of Commonly-Implemented Background Subtraction Algorithms *
}

\author{
Y. Benezeth ${ }^{1}$ \\ P.M. Jodoin ${ }^{2}$ \\ B. Emile ${ }^{1}$ \\ H. Laurent ${ }^{1}$ \\ C. Rosenberger ${ }^{3}$ \\ ${ }^{1}$ Institut PRISME \\ ${ }^{2}$ MOIVRE \\ Université d'Orléans \\ 88 boulevard Lahitolle \\ Université de Sherbrooke \\ ${ }^{3}$ GREYC, ENSICAEN \\ 2500 boulevard de l'Université \\ Université de Caen - CNRS \\ 18020 Bourges Cedex, France \\ Sherbrooke, J1K 2R1, Canada \\ 6 boulevard Maréchal Juin \\ 14000 Caen, France
}

\begin{abstract}
Locating moving objects in a video sequence is the first step of many computer vision applications. Among the various motion-detection techniques, background subtraction methods are commonly implemented, especially for applications relying on a fixed camera. Since the basic inter-frame difference with global threshold is often a too simplistic method, more elaborate (and often probabilistic) methods have been proposed. These methods often aim at making the detection process more robust to noise, background motion and camera jitter. In this paper, we present commonly-implemented background subtraction algorithms and we evaluate them quantitatively. In order to gauge performances of each method, tests are performed on a wide range of real, synthetic and semi-synthetic video sequences representing different challenges.
\end{abstract}

\section{Introduction}

For various computer vision applications, background subtraction (BS) is a "quick and dirty" way of localizing moving objects. Based on the assumption that a moving object is made of colors which differ from those in the background, typical BS methods label "in motion" every pixel at time $t$ whose color is significantly different from the ones in the background [10]. Unfortunately, a simple interframe difference with global threshold reveals itself as being sensitive to phenomena that violate the basic assumptions of BS, i.e. a rigorously fixed camera with a static noise-free background [4]. In real-life scenarios, the illumination can change (gradually or suddenly), the background may contain moving objects (waves on the water, trees shaken by the wind), the camera can jitter and so forth.

\footnotetext{
*This work was realized with the financial help of the Regional Council of Le Centre and the French Industry Ministry within the Capthom project of the Competitiveness Pole $S^{2} E^{2}$
}

In order to deal with those challenges, numerous background models and distance measures have been proposed. Those methods are (at least in theory) more robust to background instability than the basic ones. But are they really? And if they are, how much better are they? In this paper, we review commonly-implemented BS methods which we compare on various real, synthetic and semi-synthetic sequences. In section 2 , five commonly-implemented motion detection methods are described. The video dataset used to compare those methods is described in section 3 while results and conclusion are presented in sections 4 and 5.

\section{Background Subtraction Algorithms}

Although different, most BS techniques share a common framework: they make the hypothesis that the observed video sequence $I$ is made of a fixed background $B$ in front of which moving objects are observed. With the assumption that a moving object at time $t$ has a color (or a color distribution) different from the one observed in $B$, the principle of BS methods can be summarized by the following formula:

$$
\mathcal{X}_{t}(s)=\left\{\begin{array}{cc}
1 & \text { if } d\left(I_{s, t}, B_{s}\right)>\tau \\
0 & \text { otherwise }
\end{array}\right.
$$

where $\mathcal{X}_{t}$ is the motion label field at time $t$ (also called motion mask), $d$ is a distance between $I_{s, t}$ the video frame at time $t$ at pixel $s$ and $B_{s}$ the background at pixel $s ; \tau$ is a threshold. The main difference between most BS methods is how $B$ is modeled and which distance metric $d$ is being used. In the following subsections, various BS techniques are presented.

\section{Basic Motion Detection (Basic)}

The easiest way to model the background is with a grayscale/color image $B$. This image can be a picture taken in absence of moving objects and/or estimated via a temporal median filter [10]. In order to keep the background up to date, it can be iteratively updated as follows: 


$$
\boldsymbol{B}_{s, t+1}=(1-\alpha) \boldsymbol{B}_{s, t}+\alpha \cdot \boldsymbol{I}_{s, t}
$$

where $\alpha$ is an updating constant whose value ranges between 0 and 1 . Foreground pixels can be detected by thresholding various distance metrics such as:

$$
\begin{aligned}
d_{0}= & \left|I_{s, t}-B_{s, t}\right| \\
d_{1}= & \left|I_{s, t}^{R}-B_{s, t}^{R}\right|+\left|I_{s, t}^{G}-B_{s, t}^{G}\right|+\left|I_{s, t}^{B}-B_{s, t}^{B}\right| \\
d_{2}= & \left(I_{s, t}^{R}-B_{s, t}^{R}\right)^{2}+\left(I_{s, t}^{G}-B_{s, t}^{G}\right)^{2} \\
& +\left(I_{s, t}^{B}-B_{s, t}^{B}\right)^{2} \\
d_{\infty}= & \max \left\{\left|I_{s, t}^{R}-B_{s, t}^{R}\right|,\left|I_{s, t}^{G}-B_{s, t}^{G}\right|,\left|I_{s, t}^{B}-B_{s, t}^{B}\right|\right\}
\end{aligned}
$$

where exponents $R, G$ and $B$ stand for the red, green and blue channels. Note that distance $d_{0}$ operates on grayscale images.

\section{One Gaussian (1-G)}

Many authors model each background pixel with a probability density function (PDF) learned over a set of training frames. In this case, the BS problem often becomes a PDF-thresholding problem. For instance, to account for noise, some authors [9] model every background pixel with a Gaussian distribution $\eta\left(\boldsymbol{\mu}_{s, t}, \boldsymbol{\Sigma}_{s, t}\right)$ where $\boldsymbol{\mu}_{s, t}$ and $\boldsymbol{\Sigma}_{s, t}$ stand for the average background color and covariance matrix over pixel $s$ at time $t$. In this context, the distance metric can be the log likelihood:

$$
\begin{aligned}
d_{G}= & \frac{1}{2} \log \left((2 \pi)^{3}\left|\boldsymbol{\Sigma}_{s, t}\right|\right) \\
& +\frac{1}{2}\left(\boldsymbol{I}_{s, t}-\boldsymbol{\mu}_{s, t}\right) \boldsymbol{\Sigma}_{\boldsymbol{s}, \boldsymbol{t}}^{-\mathbf{1}}\left(\boldsymbol{I}_{s, t}-\boldsymbol{\mu}_{s, t}\right)^{T}
\end{aligned}
$$

or the Mahalanobis distance:

$$
d_{M}=\left|\boldsymbol{I}_{s, t}-\boldsymbol{\mu}_{s, t}\right| \boldsymbol{\Sigma}_{s, t}^{-1}\left|\boldsymbol{I}_{s, t}-\boldsymbol{\mu}_{s, t}\right|^{T}
$$

where $\mathbf{I}_{s, t}$ and $\boldsymbol{\mu}_{s, t}$ are RGB vectors and $\boldsymbol{\Sigma}_{s, t}$ is a covariance matrix. To account for illumination variations, the mean and covariance of each pixel can be iteratively updated as follows:

$$
\begin{aligned}
\boldsymbol{\mu}_{s, t+1}= & (1-\alpha) \cdot \boldsymbol{\mu}_{s, t}+\alpha \cdot \boldsymbol{I}_{s, t} \\
\boldsymbol{\Sigma}_{s, t+1}= & (1-\alpha) \cdot \boldsymbol{\Sigma}_{s, t} \\
& +\alpha \cdot\left(\boldsymbol{I}_{s, t}-\boldsymbol{\mu}_{s, t}\right)\left(\boldsymbol{I}_{s, t}-\boldsymbol{\mu}_{s, t}\right)^{T}
\end{aligned}
$$

Note that the covariance matrix can be a $3 \times 3$ matrix or can be assumed to be diagonal to reduce processing costs.

\section{Gaussian Mixture Model (GMM)}

To account for backgrounds containing animated textures (such as waves on the water or trees shaken by the wind), multimodal PDFs have been proposed. For instance, Stauffer and Grimson [8] model every pixel with a mixture of $K$ Gaussians. Thus, the probability of occurrence of a color at a given pixel $s$ is represented as:

$$
P\left(I_{s, t}\right)=\sum_{i=1}^{K} \omega_{i, s, t} . \eta\left(\boldsymbol{I}_{s, t}, \boldsymbol{\mu}_{i, s, t}, \boldsymbol{\Sigma}_{i, s, t}\right)
$$

where $\eta\left(\boldsymbol{I}_{s, t}, \boldsymbol{\mu}_{i, s, t}, \boldsymbol{\Sigma}_{i, s, t}\right)$ is the $i^{\text {th }}$ Gaussian model and $\omega_{i, s, t}$ its weight. Note that for computational reasons, as suggested by Stauffer and Grimson, we assume that the covariance matrix $\boldsymbol{\Sigma}_{i, s, t}$ is diagonal. In their method, parameters of a matched component (i.e. the Gaussian model for which $I_{s, t}$ is within 2.5 standard deviations of its mean) are updated as follows:

$$
\begin{aligned}
\omega_{i, s, t} & =(1-\alpha) \omega_{i, s, t-1}+\alpha \\
\mu_{i, s, t} & =(1-\rho) \cdot \mu_{i, s, t-1}+\rho \cdot I_{i, s, t} \\
\sigma_{i, s, t}^{2} & =(1-\rho) \cdot \sigma_{i, s, t-1}^{2}+\rho .\left(I_{i, s, t}-\mu_{i, s, t}\right)^{2}
\end{aligned}
$$

where $\alpha$ is a user-defined learning rate and $\rho$ is a second learning rate defined as $\rho=\alpha \eta\left(\boldsymbol{I}_{s, t}, \boldsymbol{\mu}_{i, s, t}, \boldsymbol{\Sigma}_{i, s, t}\right)$. The $\mu$ and $\sigma$ parameters of unmatched distributions remain the same while their weight is reduced as follows: $\omega_{i, s, t}=(1-\alpha) \omega_{i, s, t-1}$ to achieve decay. Whenever no component matches $\boldsymbol{I}_{s, t}$, the one with lowest weight is replaced by a Gaussian with mean $\boldsymbol{I}_{s, t}$, a large initial variance $\sigma_{0}$ and a small weight $\omega_{0}$. Once every Gaussian has been updated, the $K$ weights $\omega_{i, s, t}$ are normalized so they sum up to 1 . Then, the $K$ distributions are ordered based on a fitness value $\omega_{i, s, t} / \sigma_{i, s, t}$ and only the $H$ most reliable are chosen as part of the background :

$$
H=\underset{h}{\operatorname{argmin}}\left(\sum_{i=1}^{h} \omega_{i}>\tau\right)
$$

where $\tau$ is a threshold. Then, those pixels which are at more than 2.5 standard deviations away from any of those $H$ distributions are labeled "in motion".

\section{Kernel Density Estimation (KDE)}

An unstructured approach can also be used to model a multimodal PDF. In this perspective, Elgammal et al. [5] proposed a Parzen-window estimate of every background pixel:

$$
P\left(I_{s, t}\right)=\frac{1}{N} \sum_{i=t-N}^{t-1} K\left(I_{s, t}-I_{s, i}\right)
$$

where $K$ is a kernel (typically a Gaussian one) and $N$ is the number of previous frames used to estimate $P($.$) .$ When dealing with color video frames, products of onedimensional kernels can be used:

$$
P\left(\boldsymbol{I}_{s, t}\right)=\frac{1}{N} \sum_{i=t-N}^{t-1} \prod_{j=\{R, G, B\}} K\left(\frac{\left(I_{s, t}^{j}-I_{s, i}^{j}\right)}{\sigma_{j}}\right)
$$

A pixel is labeled as foreground if it is unlikely to come from this distribution, i.e. when $P\left(I_{t}\right)$ is smaller than a predefined threshold. Note that $\sigma_{j}$ can be fixed or preestimated following Elgammal et al.'s method [5].

\section{Minimum, Maximum and Maximum Inter-Frame Difference (MinMax)}

The $W^{4}$ videosurveillance system [6] uses a background model made of a minimum $m_{s}$, a maximum $M_{s}$, and a maximum of consecutive frames difference $D_{s}$. For MinMax, a pixel $s$ belongs to the background if: 


$$
\left|M_{s}-I_{s, t}\right|<\tau d_{\mu} \quad \text { or } \quad\left|m_{s}-I_{s, t}\right|<\tau d_{\mu}
$$

where $\tau$ is an user-defined threshold and $d_{\mu}$ is the mean of the largest interframe difference over pixels. Note that MinMax operates on grayscale videos only.

\section{Video Dataset}

In order to gauge performances, the five BS methods have been executed on a wide range of real, synthetic and semi-synthetic video sequences (some examples are given in figure 1). Our dataset is composed of 29 video sequences (15 real, 10 semi-synthetic and 4 synthetic). While some synthetic and semi-synthetic videos have been created by the authors, others were downloaded from the PETS2001 dataset [1], the IBM dataset [3] and the VSSN 2006 competition [2]. The semi-synthetic videos are made of synthetic foreground objects (people and cars) moving over a real background. Those videos represent both indoor (20 videos) and outdoor scenes (9 videos). Moreover, 6 videos contain animated background textures. Ground truths are easily obtained for synthetic and semi-synthetic video sequences. For real videos, the ground truth is only available on some reference images (manually annotated or provided with the dataset).

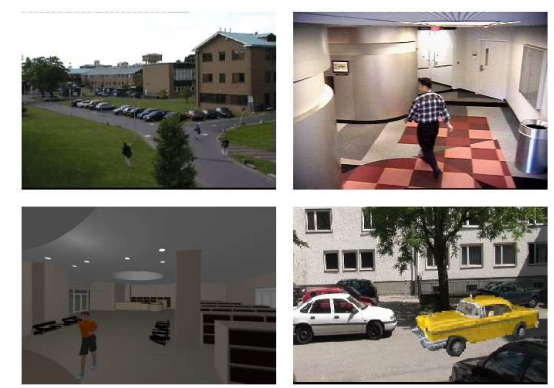

Figure 1. Snapshots from the dataset

\section{Experimental Results}

Since our dataset is made of videos representing different challenges, we divided those sequences into three categories: (1) the noise-free with perfectly fixed background sequences, (2) the multimodal sequences and (3) the noisy sequences. Each of these dataset is composed of real, synthetic and semi-synthetic video sequences. Those categories are used to evaluate and compare the different background models in section 4.2. In order to quantitatively evaluate each method, precision and recall average values are used [7]. Note that we use the $R G B$ color space and a despeckle filter is applied on every motion mask in order to eliminate groups of 4-connected foreground pixels made of less than 8 pixels.

\subsection{Influence of the Distance Measure}

As mentioned in section 2, different distance measures can be implemented in BS methods. To quantify those distance measures, the Basic method has been executed on 15 videos taken from categories (1) and (3).

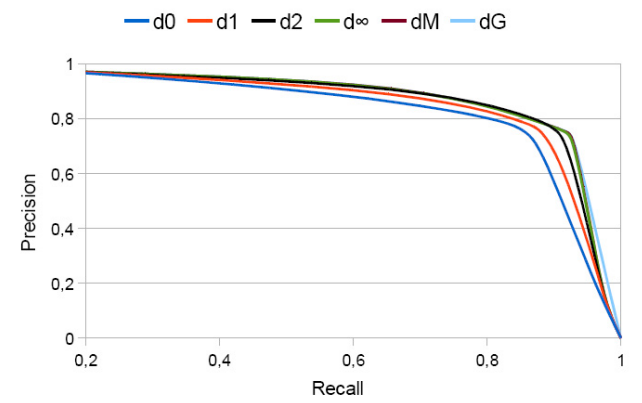

Figure 2. ROC Curves obtained for the six different distance measures

As can be seen in figure 2, the four distance measures $d_{2}, d_{\infty}, d_{M}$ and $d_{G}$ globally produced the same results, while $d_{0}$ and $d_{1}$ seem slightly less precise. Table 1 shows results obtained with a fixed precision of 0.75. A fixed precision means that every method has been tuned to produce the same amount of true positives, while the number of false positives is used to differentiate the methods. Again though, the uniform recall values underscore the fact that none of the 4 remaining distance measures outperforms the other ones.

\begin{tabular}{|c|c|c|c|c|c|c|}
\hline & $d_{0}$ & $d_{1}$ & $d_{2}$ & $d_{\infty}$ & $d_{M}$ & $d_{G}$ \\
\hline Recall & 0.88 & 0.90 & $\mathbf{0 . 9 2}$ & $\mathbf{0 . 9 2}$ & $\mathbf{0 . 9 2}$ & $\mathbf{0 . 9 2}$ \\
\hline
\end{tabular}

Table 1. Recall values with fixed precision $(0.75)$ for the six distance measures

\subsection{Evaluation of Background Models}

Every background models are compared on videos taken from the three categories previously introduced. Note that distance $d_{2}$ is used for algorithm Basic, $d_{M}$ for $1-G, K=3$ for $G M M, N=100$ and $\sigma_{j}$ is preestimated following Elgammal et al.'s method [5] for $K D E$, the learning rate $\alpha$ is fixed to $10^{-3}$ for Basic, $1-G$ and $G M M$. Others parameters have been tuned to fix the precision value to 0.75 or 0.5 .

Test 1: Evaluation on Noise-Free with Perfectly Static Background Videos

The BS algorithms presented in section 2 were executed on noise-free video sequences exhibiting a rigorously static background. A total of 15 videos (both indoor and outdoor) have been retained. Results with precision 
fixed to 0.75 are presented in table 2. Interestingly, the results are globally homogeneous. Algorithms Basic, $1-G$ and $G M M$ are, to all practical purposes, equivalent while algorithms $K D E$ and MinMax show less effectiveness. The Basic algorithm is thus well suited for sequences which do not contain specific difficulties. This is a rather interesting observation since the Basic approach is, by far, the fastest and simplest method of all.

\section{Test 2: Evaluation on Multimodal Videos}

The second test aims at evaluating the robustness of every BS methods to animated background textures. Here, 6 video sequences exhibiting strong background motion have been used. Results with precision fixed to 0.5 are presented in table 2 .

As one would expect, the Basic and MinMax methods are strongly penalized by this test. On the other hand, results obtained with the $1-G$ method are surprisingly good. This can be explained by the fact that the $1-G$ threshold is locally weighted by a covariance matrix which compensates well some background instabilities. Thanks to their multimodal shape, the $K D E$ and GMM methods produced the most accurate results. Figure 3 presents examples of detection on a semi-synthetic video with a strongly animated background.

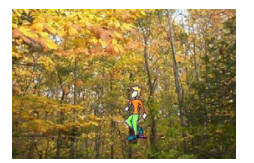

Original image

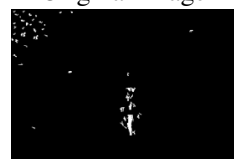

MinMax

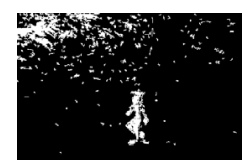

Basic

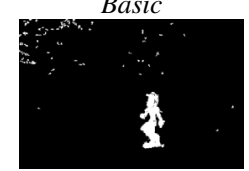

$G M M$

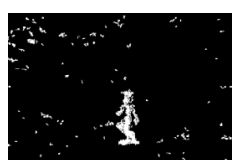

$1-G$

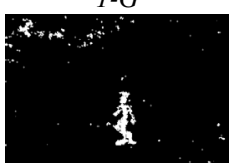

$K D E$
Figure 3. Results for different BS algorithms on a semi-synthetic multimodal video

\section{Test 3: Evaluation on Noisy Videos}

The third test aims at evaluating the influence of noise. Here, 15 videos corrupted with strong additive Gaussian noise are used for testing. Results with precision fixed to 0.75 are presented in table 2 . As can be seen, methods $1-G, K D E$ and $G M M$ produced good, yet homogeneous recall values while the MinMax method does not seem to be well suited for noisy videos. This may be explained by the fact that the MinMax threshold (which is global) depends on the maximum interframe difference (which is large for noisy videos). Moreover, the MinMax method works on grayscale sequences and thus ignores color information. For the Basic method, its global threshold significantly penalizes the performances.

\begin{tabular}{|c|c|c|c|c|c|}
\hline & Basic & $1-\mathrm{G}$ & KDE & MinMax & GMM \\
\hline test 1 & 0.92 & 0.92 & 0.88 & 0.88 & $\mathbf{0 . 9 3}$ \\
\hline test 2 & 0.55 & 0.76 & $\mathbf{0 . 8 4}$ & 0.48 & 0.79 \\
\hline test 3 & 0.62 & $\mathbf{0 . 7 7}$ & 0.75 & 0.28 & 0.76 \\
\hline
\end{tabular}

Table 2. Recall values for different video sequences. Precision is fixed to 0.75 for test 1 and test 3 and to 0.5 for test 2 .

\section{Conclusion}

In this paper, we reviewed and evaluated commonlyimplemented BS algorithms. Evaluation, performed on a large video dataset made of various real, synthetic and semi-synthetic video sequences, allows us to draw two conclusions. First, no method outperforms the other ones on every video category. Consequently, the choice of a BS method shall be motivated more by the content of the scene than the model itself. For instance, when dealing with videos respecting the fundamental BS assumption (a fixed camera with a static noise-free background), a basic BS implementation is well suited and no better results can be expected by other, yet more complicated, methods. Secondly, the $1-G, G M M$ and $K D E$ methods are the most reliable over noisy sequences. Thirdly, for videos containing significant background motion, methods with a global threshold (namely the Basic and MinMax methods) are significantly penalized while $G M M$ and $K D E$ show good robustness.

Our future work will focus on the robustness of these BS methods to bursty background motion and to corrupted training sequences. We will also compare the amount of memory and processing power those methods require.

\section{References}

[1] www.cvg.cs.rdg.ac.uk/PETS2001.

[2] http://imagelab.ing.unimore.it/vssn06.

[3] L. Brown, A. Senior, Y. Tian, J. Vonnel, A. Hampapur, C. Shu, H. Merkl, and M. Lu. Performance evaluation of surveillance systems under varying conditions. in IEEE Proc. PETS Workshop, 2005.

[4] S. Cheung and C. Kamath. Robust techniques for background subtraction in urban traffic video. in Proc. of the VCIP, 2004.

[5] A. Elgammal, D. Harwood, and L. Davis. Non-parametric model for background subtraction. in ECCV, 2000.

[6] I. Haritaoglu, D. Harwood, and L. Davis. W4-real time detection and tracking of people and their parts. in IEEE Trans. PAMI, 2000.

[7] A. Prati, I. Mikic, M. Trivedi, and R. Cucchiara. Detecting moving shadows: Algorithms and evaluation. in IEEE Trans. PAMI, 2003.

[8] C. Stauffer and W. Grimson. Adaptive background mixture models for real-time tracking. in IEEE Proc. CVPR, 1999.

[9] C. Wren, A. Azarbayejani, T. Darrell, and A. Pentland. Pfinder: Real-time tracking of the human body. in IEEE Trans. PAMI, 1997.

[10] Q. Zhou and J. Aggarwal. Tracking and classifying moving objects from video. in IEEE Proc. PETS Workshop, 2001. 